\title{
On a new measure on fractals
}

\author{
Alireza K Golmankhaneh¹ and Dumitru Baleanu'2,3,4*
}

${ }^{\text {*Correspondence: }}$

dumitru@cankaya.edu.tr

${ }^{2}$ Department of Chemical and

Materials Engineering, Faculty of

Engineering, King Abdulaziz

University, P.O. Box 80204, Jeddah,

21589, Saudi Arabia

${ }^{3}$ Department of Mathematics and

Computer Science, Çankaya

University, Ankara, 06530, Turkey

Full list of author information is

available at the end of the article

\begin{abstract}
Fractals are sets whose Hausdorff dimension strictly exceeds their topological dimension. The algorithmic Riemannian-like method, $F^{\alpha}$-calculus, has been suggested very recently. Henstock-Kurzweil integral is the generalized Riemann integral method by using the gauge function. In this paper we generalize the $F^{\alpha}$-calculus as a fractional local calculus that is more suitable to describe some physical process. We introduce the new measure using the gauge function on fractal sets that gives a finer dimension in comparison with the Hausdorff and box dimension. Hilbert $F^{\alpha}$-spaces are defined. We suggest the self-adjoint $F^{\alpha}$-differential operator so that it can be applied in the fractal quantum mechanics and on the fractal curves.
\end{abstract}

Keywords: fractal measure; fractal calculus; fractal curve

\section{Introduction}

Fractal geometry is used to describe real objects such as trees, lightning, river meanders and coastlines. We known that the Euclidean geometry is the approximate geometry applied in the real world. In the sense of Mandelbrot, a fractal set is the one whose Hausdorff dimension strictly exceeds the topological dimension [1-4]. Therefore, the calculus on fractals leads to better understanding the description of various real world models from science and engineering. Researchers have constructed analysis on fractals by using different approaches. For example, the fractional calculus has been applied to explain the supper- and sub-diffusion in physics as well as many other non-local phenomena. The fractional derivatives are non-local and they have memory property [5-8]. Recently, the fractional local calculus has been defined and applied in various fields [9-13]. The calculus on fractals which is Riemannian-like was suggested in [14, 15]. Meanwhile, it was generalized and applied in Newtonian, Lagrange and Hamilton mechanics. The Maxwell and the Schrödinger equations were expanded on fractal imbedding in $R^{3}$ [16-19]. Moreover, the measure theoretical approaches were used to create a calculus on fractals [20, 21]. In recent years, the calculus on fractals became an interesting and powerful tool for researchers. Taking into account the motivation presented above, in this manuscript we suggest a new measure on fractals. The relative extrema in $F^{\alpha}$-calculus are discussed. The Hilbert $F^{\alpha}$-spaces and $F^{\alpha}$-self-adjoint differential operator are defined.

The plan of the paper is given below.

In Section 2 we review the definitions of measurable sets and Hausdorff measure. In Section 3 a new measure on fractal sets is introduced. We introduce the relative extrema condition of the $F^{\alpha}$-calculus in Section 4 . Section 5 presents the Hilbert spaces in an $F^{\alpha}$ -

○2013 Golmankhaneh and Baleanu; licensee Springer. This is an Open Access article distributed under the terms of the Creative Commons Attribution License (http://creativecommons.org/licenses/by/2.0), which permits unrestricted use, distribution, and reproduction in any medium, provided the original work is properly cited. 
space. In Section 6 the $F^{\alpha}$-self-adjoint differential operator is defined. Section 7 is devoted to conclusions.

\section{Preliminaries}

As it is known, measure theory extends the concept of length for an arbitrary subset of the real line. Thus, a measure is the generalization of the concepts of length, area and volume [23]. Lebesgue introduced a measure that leads to a calculus on $R^{n}$. In this section we review the basic tools used in this paper. Firstly, we recall the definition of measurable sets such as Borel sets. Secondly, the Hausdorff measure is reviewed so that it is useful for analysis on fractals [23]. We notice that the Lebesgue measure is zero on the Cantor set as a fractal subset of the real line. More details about this subject can be found in the papers [9-15].

\subsection{Measurable sets}

Let $R$ and $P(R)$ be all subsets or a power set of $R$. Boolean algebra $\Re$ of the sets in $P(R)$ has the following conditions $[2,3,22,23]$ :

(I) $A, B \in \mathfrak{R} \Rightarrow A \cup B \in \mathfrak{R}$,

(II) $A \in \mathfrak{R} \Rightarrow A^{c}=R-A \in \mathfrak{R}$,

(III) $A, B \in \mathfrak{R} \Rightarrow A \cap B \in \mathfrak{R}$,

where $\mathfrak{R}$ are subsets of a set $P(R)$. An algebra $\Re$ is $\sigma$-algebra if it is closed under countable union of sets.

$\mu$ is a measure on $\mathfrak{R}$ if for a number of subsets of $\mathfrak{R}$, we have

$$
\begin{aligned}
& \mu(\emptyset)=0 ; \\
& \mu(A) \leq \mu(B) \quad \text { if } A \subset B .
\end{aligned}
$$

If $A_{1}, A_{2}, \ldots$ is a countable sequence of sets, thus

$$
\mu\left(\bigcup_{i=1}^{\infty}\right) \leq \sum_{i=1}^{\infty} \mu\left(A_{i}\right)
$$

equality is for the disjoint Borel sets.

\subsection{Hausdorff measure}

Suppose that the $(R, \rho)$ is a metric space and $K \subset R$, thus the diameter of $K$ is [2, 3, 22, 23]

$$
\operatorname{diam} K:=\sup \{\rho(x, y) \mid x, y \in K\} .
$$

Consider $Z$ to be a subset of $R$, then we conclude that

$$
H_{\delta}^{d}(Z)=\inf \left\{\sum_{i=1}^{\infty}\left(\operatorname{diam} K_{i}\right)^{d}: \bigcup_{i=1}^{\infty} K_{i} \supseteq Z, \operatorname{diam} K_{i}<\delta\right\}
$$

where $\delta>0$ and infimum is upon all countable covers of $Z$ with the condition $K_{i} \subset R$ and $\operatorname{diam} K_{i}<\delta$. The $H_{\delta}^{d}(S)$ is decreasing, so $\lim _{\delta \rightarrow 0} H_{\delta}^{d}(S)$ exists. Then the following

$$
H^{d}(S):=\sup _{\delta>0} H_{\delta}^{d}(S)=\lim _{\delta \rightarrow 0} H_{\delta}^{d}(S)
$$


is called Hausdorff measure on fractal sets [2, 3, 22, 23]. In this definition, the covering sets can be open or closed, and they will yield the same measure.

\subsection{Generalized Hausdorff measure}

We review the fractional measure introduced on fractional sets $[9,10]$.

The fractional sets are defined as follows:

$$
\begin{aligned}
& N^{\alpha}=\left\{1^{\alpha}, 2^{\alpha}, \ldots\right\}, \quad Z^{\alpha}=\left\{0^{\alpha}, \pm 1^{\alpha}, \pm 2^{\alpha}, \ldots\right\}, \\
& Q^{\alpha}=\left\{m^{\alpha}=\left(\frac{p}{q}\right)^{\alpha}: p, q \in Z q \neq 0\right\}, \quad 0<\alpha<1, \\
& \Im^{\alpha}=\left\{m^{\alpha} \neq\left(\frac{p}{q}\right)^{\alpha}: p, q \in Z q \neq 0\right\}, \quad 0<\alpha<1, \\
& R^{\alpha}=\Im^{\alpha} \cup Q^{\alpha} .
\end{aligned}
$$

The diameter of a non-empty fractional set $E$, the subset of a fractional set, is given by

$$
|E|:=\sup \left\{|x-y|^{\alpha}, x^{\alpha}, y^{\alpha} \in E\right\} .
$$

If $\left\{F_{i}\right\}$ is a countable collection of subsets of $R^{\alpha}$ such as $E \subset \bigcup_{i=1} d F_{i}$ and $0<\left|F_{i}\right|<\delta^{\alpha}$, then $\left\{F_{i}\right\}$ is $\delta^{\alpha}$-cover sets $[9,10]$. The $\alpha$-dimensional generalized Hausdorff measure is suggested as follows:

$$
H^{\alpha}(E)=\lim _{\delta^{\alpha} \rightarrow 0} \inf \left\{\sum_{i=1}\left|F_{i}\right|: F_{i} \text { is } \delta^{\alpha} \text { cover of } E\right\} .
$$

In the following sections, we present the main results of our manuscript.

\section{A new measure on fractals}

In this section we write the definition of a flag function. By using the gauge function, the generalized mass function is defined so that it can be used for a wider class of functions on fractal sets. Let $F$ be a subset of the real line and a fractal. The flag function for a set $F$ has the form

$$
\theta(F, I)= \begin{cases}1 & \text { if } F \cap I \neq \emptyset \\ 0 & \text { otherwise }\end{cases}
$$

where $I=[a, b]$ denotes an interval in $R$, and $P=\left\{I_{i}, i=1, \ldots, n\right\}=\left\{I_{i}\right\}_{i=1}^{n}$ is a tagged partition $[22,23]$. Let $\delta(t)$ be a gauge function on $I$. So, we say that $\dot{P}$ is $\delta(t)$-fine if

$$
I_{i} \subseteq\left[t_{i}-\delta\left(t_{i}\right), t_{i}+\delta\left(t_{i}\right)\right]
$$

Let us assume that $I=[a, b]$ is a nonempty compact interval.

Let $F$ be a subset of $I=[a, b]$. Let $\dot{P}$ be a $\delta$-fine partition. $\sigma_{*}^{\alpha}[F, I]$ is defined as

$$
\sigma_{*}^{\alpha}[F, I]=\sum_{i=1}^{n} \frac{\left(x_{i}-x_{i-1}\right)^{\alpha}}{\Gamma(\alpha+1)} \theta\left(F,\left[x_{i-1}, x_{i}\right]\right)
$$

where $a<b$ and $0<\alpha \leq 1$. 
Given the gauge $\delta(t)$ on $I=[a, b]$ and $a<b$, let $\dot{P}$ be a $\delta$-fine partition. The coarse grained mass * $\gamma_{\delta}^{\alpha}(F, a, b)$ of $F \cap[a, b]$ is given by

$$
{ }^{*} \gamma_{\delta(t)}^{\alpha}(F, a, b)=\inf _{|\dot{P}|<\sup \left\{\delta\left(t_{i}\right) ; t_{i} \in\left[x_{i-1}, x_{i}\right]\right\}} \sigma_{*}^{\alpha}[F, I]
$$

where $|\dot{P}|=\max _{1 \leq i \leq n}\left(x_{i}-x_{i-1}\right)$. We take the infimum over all $\delta$-fine partitions of $I$.

The mass function * $\gamma^{\alpha}(F, a, b)$ is defined as follows:

$$
{ }^{*} \gamma^{\alpha}(F, a, b)=\lim _{\sup \left\{\delta\left(t_{i}\right): t_{i} \in\left[x_{i-1}, x_{i}\right]\right\} \rightarrow 0}{ }^{*} \gamma_{\delta(t)}^{\alpha}(F, a, b) .
$$

${ }^{*} \gamma_{\delta}^{\alpha}$ is increasing with $\delta$, then we can define $[19,22,23]$

$$
{ }^{*} \gamma_{0}^{\alpha}(F, a, b)=\lim _{\sup \left\{\delta\left(t_{i}\right) ; t_{i} \in\left[x_{i-1}, x_{i}\right]\right\} \rightarrow 0} * \gamma_{\delta(t)}^{\alpha}(F, a, b),
$$

if it exists. We mention that ${ }^{*} \gamma_{0}^{\alpha}$ is not a measure because countable additivity fails. We define the new measure arising from the gauge function and denote it by $\mathbf{G}^{\alpha}(F, a, b)$. Let ${ }^{*} \gamma^{\alpha}(F, a, b)$ be a set of functions over Borel sets $F \subset R$ as

$$
\mathbf{G}^{\alpha}(F, a, b)=\inf \left\{\sum_{i=1}^{\infty}{ }^{*} \gamma_{0}^{\alpha}\left(F_{i}, a, b\right) F \subset \bigcup_{i=1}^{\infty} F_{i}\right\}
$$

where $F_{i}$ are countable closed covers of the set $F$. Here, we are taking infimum over all closed covers of $F$. Now, as a measure $\mathbf{G}^{\alpha}(F, a, b)$, we check the properties as follows.

Properties Now, we check all the properties for the new measure, namely:

(1) It is clear that the measure of a null set is zero. Since ${ }^{*} \gamma_{\delta}^{\alpha}(\emptyset, a, b)=0$, then $\mathbf{G}^{\alpha}(\emptyset, a$, b) $=0$.

(2) If $A \subseteq B$ and $B \subseteq \bigcup_{n \in N} E_{n}$, where $E_{n}$ is a measurable set, then the set $A$ is also $A \subseteq$ $\bigcup_{n \in N} E_{n}$. Subsequently, we have

$$
{ }^{*} \gamma_{\delta}^{\alpha}(A, a, b) \leq{ }^{*} \gamma_{\delta}^{\alpha}(B, a, b)
$$

Therefore we conclude that

$$
\mathbf{G}^{\alpha}(A, a, b) \leq \mathbf{G}^{\alpha}(B, a, b) .
$$

(3) Now, we demonstrate that if $A=\bigcup_{i \in N} A_{i}$, where $A_{i}$ is a sequence of measurable sets, then $\mathbf{G}^{\alpha}(A, a, b) \leq \sum_{i=1}^{\infty} \mathbf{G}^{\alpha}\left(A_{i}, a, b\right)$.

Proof If $\sum_{i=1} \mathbf{G}^{\alpha}\left(A_{i}, a, b\right)$ diverges, then there is nothing to prove. So, we assume that $\sum_{i=1} \mathbf{G}^{\alpha}\left(A_{i}, a, b\right)<\infty$. Let $\epsilon>0$, then for each $i$ there exist sets $E_{n i}, n \in N$, such that $A_{i} \subseteq \bigcup_{n=1} E_{n i}$ and

$$
\sum_{n=1}^{*} \gamma_{0}^{\alpha}\left(E_{n i}, a, b\right)<\mathbf{G}^{\alpha}\left(A_{i}, a, b\right)+\epsilon / 2^{i}
$$


Since $A \subseteq \bigcup_{i} \bigcup_{n} E_{n i}$, we have

$$
\begin{aligned}
\mathbf{G}^{\alpha}(A, a, b) & \leq \sum_{i} \sum_{n} \gamma_{0}^{\alpha}\left(E_{n i}, a, b\right)<\sum_{i}\left(\mathbf{G}^{\alpha}\left(A_{i}, a, b\right)+\frac{\epsilon}{2^{i}}\right) \\
& =\sum_{i} \mathbf{G}^{\alpha}\left(A_{i}, a, b\right)+\epsilon .
\end{aligned}
$$

Since this holds for any $\epsilon>0$, therefore we obtain

$$
\mathbf{G}^{\alpha}(A, a, b) \leq \sum_{i=1} \mathbf{G}^{\alpha}\left(A_{i}, a, b\right)
$$

(4) If $\left\{A_{i}\right\}$ is a countable family of disjoint measurable sets such that $A=\bigcup_{i} A_{i}$, then

$$
\mathbf{G}^{\alpha}\left(\bigcup_{i} A_{i}, a, b\right)=\sum_{i} \mathbf{G}^{\alpha}\left(A_{i}, a, b\right) .
$$

Proof Let $A_{1}, A_{2}, \ldots, A_{n}$ be disjoint measurable sets. Noticing that the set $A_{1}$ is measurable, if one picks out a test set as $T=A_{1} \cup A_{2} \cup \cdots \cup A_{n}$, therefore, we have

$$
\mathbf{G}^{\alpha}\left(A_{1}, a, b\right)+\mathbf{G}^{\alpha}\left(A_{2} \cup A_{3}, \ldots, A_{n}, a, b\right)=\mathbf{G}^{\alpha}\left(A_{1} \cup A_{2} \cup \cdots \cup A_{n}, a, b\right) .
$$

Recalling that $A_{2}$ is measurable, by choosing a test set $T=A_{2} \cup A_{3} \cup \cdots \cup A_{n}$, we have

$$
\mathbf{G}^{\alpha}\left(A_{2}, a, b\right)+\mathbf{G}^{\alpha}\left(A_{3} \cup A_{4}, \ldots, A_{n}, a, b\right)=\mathbf{G}^{\alpha}\left(A_{2} \cup A_{3} \cup \cdots \cup A_{n}, a, b\right) .
$$

In a finite number of steps, we conclude that

$$
\mathbf{G}^{\alpha}\left(\bigcup_{i}^{n} A_{i}, a, b\right)=\sum_{i=1}^{n} \mathbf{G}^{\alpha}\left(A_{i}, a, b\right) .
$$

Notice that $\left\{A_{i}\right\}$ is a countable family of disjoint measurable sets. For each $n$, we have

$$
\mathbf{G}^{\alpha}\left(\bigcup_{i}^{\infty} A_{i}, a, b\right) \geq \mathbf{G}^{\alpha}\left(\bigcup_{i}^{n} A_{i}, a, b\right)=\sum_{i=1}^{n} \mathbf{G}^{\alpha}\left(A_{i}, a, b\right) .
$$

Hence, we obtain the following inequality:

$$
\mathbf{G}^{\alpha}\left(\bigcup_{i}^{\infty} A_{i}, a, b\right) \geq \sum_{i=1}^{\infty} \mathbf{G}^{\alpha}\left(A_{i}, a, b\right)
$$

In view of (14)-(18), the proof is complete.

Finally, we define $\beta$-dimension as it is given below

$$
\operatorname{dim}_{\beta} F=\inf \left\{\alpha: \mathbf{G}^{\alpha}(F, a, b)=0\right\}=\sup \left\{\alpha: \mathbf{G}^{\alpha}(F, a, b)=\infty\right\} .
$$




\section{Relative extrema in $F^{\alpha}$-calculus}

In the rest of the paper, we generalize the $F^{\alpha}$-calculus on a fractal subset of the real-line [14]. We recall that the extrema of a function are the values that the function is either maximum or minimum. Suppose that $f: I \rightarrow R$ and $\operatorname{Sch}(f)$ contained in $F$, which is $\alpha$ perfect [14], is said to have a relative maximum / relative minimum at $c \in I$ if there exists a neighborhood $V=V_{\delta}(c)$ of $c$ such that $f(x) \leq f(c)$ [respectively $f(c) \leq f(x)$ ] for all $x$ in $V_{\delta} \cap F$. We say that $f$ has a relative extremum at $c \in I$ if it has either a relative maximum or a relative minimum at $c[19]$.

Analogue of Fermat's theorem in $F^{\alpha}$-calculus Let $f: I \rightarrow R$ be a function such that $S c h(f)$ is contained in $F$ which is an $\alpha$-perfect set. Suppose that $c \in I$ is a relative extrema off. Iff is $F^{\alpha}$-differentiable at c, then $D_{F}^{\alpha} f(x)=0$ [14].

Proof Let $c$ be a relative maximum. Then there is $\delta>0$ such that $V_{\delta}(c)=(c-\delta, c+\delta) \subset I$ for all $x \in V_{\delta} \cap F$, we have $f(x) \leq f(c)$.

If $D_{F}^{\alpha} f(x)>0$ so that there exists a neighborhood $V_{\delta}(c) \subseteq I$ such that

$$
\frac{f(x)-f(c)}{S_{F}^{\alpha}(x)-S_{F}^{\alpha}(c)}>0 \quad \text { for } x \in V_{\delta} \cap F
$$

If $x \in V_{\delta} \cap F$ and $x>c$, then we have

$$
f(x)-f(c)=\left(S_{F}^{\alpha}(x)-S_{F}^{\alpha}(c)\right) \cdot \frac{f(x)-f(c)}{S_{F}^{\alpha}(x)-S_{F}^{\alpha}(c)}>0 .
$$

However, this contradicts that $f$ has a relative maximum at $c$. Therefore, we cannot have $D_{F}^{\alpha} f(x)>0$. Similarly, we cannot have $D_{F}^{\alpha} f(x)<0$. Hence, we must have $D_{F}^{\alpha} f(x)=0$.

Remark A similar proof applies if $c$ is a relative minimum.

\section{Hilbert spaces in $F^{\alpha}$-calculus}

The Hilbert space is a vector space equipped with inner product such that every Cauchy sequence has limit that belongs to the vector space. In most cases in the quantum mechanics, we need to construct the Hilbert spaces. Therefore, in this section, we introduce the analogues of Hilbert spaces on a fractal set subset of the real line [14, 19]. Consider the space of functions, $f:[a, b] \rightarrow R$ and $\operatorname{Sch}(f)$ is an $\alpha$-perfect set[14]. Thus, we have the following:

$$
\int_{a}^{b}|f(x)|^{2} d_{F}^{\alpha} x<\infty
$$

This space is called a square $F^{\alpha}$-integrable function and it is denoted by $L_{2}^{\alpha}$. If $f, g \in L_{2}^{\alpha}$, then $a f+b g \in L_{2}^{\alpha}$, where $a, b \in R$. $L_{2}^{\alpha}$ is a complete vector space. We introduce the inner product in an $F^{\alpha}$-space as

$$
\langle f \mid g\rangle=\int_{a}^{b} f(x) g(x) d_{F}^{\alpha} x
$$


where $f, g \in L_{2}^{\alpha}$. This inner product gives us a positive definite norm for each vector such that

$$
\|f\|=[\langle f \mid f\rangle]^{1 / 2},
$$

$\|f\|=0$ if only if $f=0$. A distance between two functions is given by

$$
d(f, g)=\|f-g\| .
$$

This inherits from the metric space $L_{2}^{\alpha}$ since this metric space is complete with respect to the metric defined in Eq. (28). Accordingly, $L_{2}^{\alpha}$ is an $F^{\alpha}$-Hilbert space, which will also be denoted by $\mathbb{H}^{\alpha}$. A system of functions $\left\{f_{n}(x)\right\}$ in $\mathbb{H}^{\alpha}$ is said to be an orthogonal system if

$$
\int_{a}^{b} f_{n}(x) f_{m}(x) d_{F}^{\alpha} x=\delta_{n, m}
$$

Here, $\delta_{n, m}$ is the Kronecker delta. Let $\left\langle f_{n}(x)\right\rangle$ be a sequence of functions which belongs to $L_{2}^{\alpha}$. Then there exists a function $f(x) \in L_{2}^{\alpha}$ with the following property:

$$
\lim _{n \rightarrow \infty} \int_{a}^{b}\left|f_{n}(x)-f(x)\right|^{2} d_{F}^{\alpha} x=0
$$

Equivalently, one can say that $f_{n}(x)$ approaches $f(x)$ if for every $\epsilon>0$, there exists a number $n_{0}>0$ such that

$$
\left\|f_{n}(x)-f(x)\right\|<\epsilon \quad \text { whenever } n>n_{0} .
$$

The sequence of functions $\left\langle f_{n}(x)\right\rangle$ belonging to $L_{2}^{\alpha}$ is called a Cauchy sequence if

$$
\lim _{\substack{m \rightarrow \infty \\ n \rightarrow \infty}} \int_{a}^{b}\left|f_{m}(x)-f_{n}(x)\right|^{2} d_{F}^{\alpha} x=0
$$

In other words, for a given $\epsilon>0$, there exists a number $n_{0}>0$ such that

$$
\left\|f_{m}-f_{n}\right\|=\int_{a}^{b}\left|f_{m}(x)-f_{n}\right|^{2} d_{F}^{\alpha} x<\epsilon \quad \text { whenever } m>n_{0}, n>n_{0} .
$$

Equation (33) means that every Cauchy sequence on a fractal set has limit belonging to the vector space. Finally, we have complied with all the conditions for a Hilbert space in $F^{\alpha}$-calculus.

\section{Self-adjoint $F^{\alpha}$-differential operator}

It is interesting to note that a second-order differential operator can be written in a SturmLiouville form if it is self-adjoint. In quantum mechanics, the eigenvalues and eigenvectors are the energy and the wave function, respectively. So, by extending the $F^{\alpha}$-calculus, namely defining an $F^{\alpha}$-differential operator, we gain the definition and the condition for the analogous Hilbert operator on a fractal set. In this section we present the self-adjoint operators involving the fractional local derivative on fractals. 
Suppose that $\mathbf{L}$ is a fractional local differential operator in the form

$$
\mathbf{L} f(x)=p_{0}(x)\left(D_{F}^{\alpha}\right)^{2} f(x)+p_{1}(x) D_{F}^{\alpha} f(x)+p_{2}(x) f(x)
$$

Now, we would like to calculate

$$
\begin{aligned}
\langle f \mid \mathbf{L} f\rangle & =\int_{a}^{b} f(x) \mathbf{L} f(x) d_{F}^{\alpha} x \\
& =\int_{a}^{b} f(x)\left\{p_{0}(x)\left(D_{F}^{\alpha}\right)^{2} f(x)+p_{1}(x) D_{F}^{\alpha} f(x)+p_{2}(x) f(x)\right\} d_{F}^{\alpha} x .
\end{aligned}
$$

By using integration by parts [14] once or twice, we conclude that

$$
\begin{aligned}
\langle f \mid \mathbf{L} f\rangle= & {\left.\left[f(x)\left(p_{1}(x)-D_{F}^{\alpha} p_{0}(x)\right) f(x)\right]\right|_{x=a} ^{x=b} } \\
& +\int_{a}^{b}\left\{\left(D_{F}^{\alpha}\right)^{2}\left[p_{0}(x) f(x)\right]-D_{F}^{\alpha}\left[p_{1}(x) f(x)\right]+p_{2}(x) f(x)\right\} f(x) d_{F}^{\alpha} x .
\end{aligned}
$$

Comprising the integrals in Eqs. (36) and (35), we get

$$
f(x)\left(\left(D_{F}^{\alpha}\right)^{2} p_{0}(x)-D_{F}^{\alpha} p_{1}(x)\right) f(x)+2 f(x)\left(D_{F}^{\alpha} p_{0}(x)-p_{1}(x)\right) D_{F}^{\alpha} f(x)=0
$$

and

$$
D_{F}^{\alpha} p_{0}(x)=p_{1}(x)
$$

Then, we finally conclude that

$$
\begin{aligned}
\overline{\mathbf{L}}[f(x)]= & \left(D_{F}^{\alpha}\right)^{2}\left[p_{0}(x) f(x)\right]-D_{F}^{\alpha}\left[p_{1}(x) f(x)\right]+p_{2}(x) f(x) \\
= & p_{0}(x)\left(D_{F}^{\alpha}\right)^{2} f(x)+\left(2 D_{F}^{\alpha} p_{0}(x)-p_{1}(x)\right) D_{F}^{\alpha} f(x) \\
& +\left(\left(D_{F}^{\alpha}\right)^{2} p_{0}(x)-D_{F}^{\alpha} p_{1}(x)+p_{2}(x)\right) f(x) .
\end{aligned}
$$

When $\overline{\mathbf{L}}[f(x)]=\mathbf{L}[f(x)]$, the operator is said to be $F^{\alpha}$-self-adjoint operator [19]. It is clear that every $F^{\alpha}$-self-adjoint operator can be written as an analogous Sturm-Liouville equation on fractal sets.

\section{Conclusion}

Researchers are trying to create new calculus on fractals by using some different approaches which are algorithmic or not. Since the fractals generated are different, the measures on them also vary from one to another. The gauge function is used to generalize the Riemann integral for a wider class of functions. We defined a new measure on fractals by using this function. This new measure can be used to define an integral on fractals. Meanwhile, the $F^{\alpha}$-calculus on fractals was generalized by given the relative extrema condition. Moreover, the Hilbert $F^{\alpha}$-space is constructed for potential applications on fractal quantum mechanics and the self-adjoint $F^{\alpha}$-differential operator is defined on fractal sets. 


\section{Competing interests}

The authors declare that they have no competing interests.

\section{Authors' contributions}

All authors read and approved the final manuscript.

\section{Author details}

'Department of Physics, Urmia Branch, Islamic Azad University, Urmia, 969, Iran. ${ }^{2}$ Department of Chemical and Materials Engineering, Faculty of Engineering, King Abdulaziz University, P.O. Box 80204, Jeddah, 21589, Saudi Arabia. ${ }^{3}$ Department of Mathematics and Computer Science, Çankaya University, Ankara, 06530, Turkey. ${ }^{4}$ Institute of Space Sciences, P.O. Box MG-23, Magurele-Bucharest, R 76900, Romania.

\section{Acknowledgements}

One of the authors (AKG) would like to thank Professor AD Gangal for useful discussion on this topic during the period of time he was in Pune University.

Received: 27 August 2013 Accepted: 13 September 2013 Published: 09 Nov 2013

\section{References}

1. Mandelbrot, BB: The Fractal Geometry of Nature. Macmillan (1983)

2. Falconer, KJ: Fractal Geometry: Mathematical Foundations and Applications. Wiley, New York (2007)

3. Falconer, KJ, Falconer, KJ: Techniques in Fractal Geometry. Wiley, Chichester (1997)

4. Edgar, GA: Integral, Probability, and Fractal Measures. Springer, Berlin (1998)

5. Samko, SG, Kilbas, AA, Marichev, Ol: Fractional Integrals and Derivatives and Some of Their Applications. Science and Technical (1987)

6. Hilfer, R: Applications of Fractional Calculus in Physics. Word Scientific, Singapore (2000)

7. Miller, KS, Ross, B: An introduction to the fractional calculus and fractional differential equations (1993)

8. Golmankhaneh, AK: Investigations in Dynamics: With Focus on Fractional Dynamics. LAP Lambert Academic Publishing, Saarbrucken (2012)

9. Yang, X-J: Advanced Local Fractional Calculus and Its Applications. World Science, New York (2012)

10. Yang, X-J: Local Fractional Functional Analysis and Its Applications. Asian Academic Publisher Limited, Hong Kong (2011)

11. Kolwankar, KM, Gangal, AD: Local fractional Fokker-Planck equation. Phys. Rev. Lett. 80, 214 (1998)

12. Kolwankar, KM, Gangal, AD: Local fractional calculus: a calculus for fractal space-time. In: Fractals, pp. 171-181. Springer, London (1999)

13. Babakhani, A, Daftardar-Gejji, V: On calculus of local fractional derivatives. J. Math. Anal. Appl. 270, 66 (2002)

14. Parvate, A, Gangal, AD: Calculus on fractal subsets of real line I. Formulation. Fractals 17(01), 53-81 (2009)

15. Parvate, A, Gangal, AD: Calculus on fractal subsets of real line II. Conjugacy with ordinary calculus. Fractals 19(03), 271-290 (2011)

16. Golmankhaneh, AK, Golmankhaneh, AK, Baleanu, D: Lagrangian and Hamiltonian mechanics on fractals subset of real-line. Int. J. Theor. Phys. 52, 4210-4217 (2013)

17. Golmankhaneh, AK, Golmankhaneh, AK, Baleanu, D: About Maxwell's equations on fractal subsets of $R^{3}$. Cent. Eur. J. Phys. 11, 863-867 (2013)

18. Golmankhaneh, AK, Fazlollahi, V, Baleanu, D: Newtonian mechanics on fractals subset of real-line. Rom. Rep. Phys. 65, 84-93 (2013)

19. Golmankhaneh, AK: Investigation in dynamics: with focus on fractional dynamics and application to classical and quantum mechanical processes. Ph.D. Thesis, submitted to University of Pune, India (2010)

20. Kigami, J: Analysis on Fractals, vol. 143. Cambridge University Press, Cambridge (2001)

21. Strichartz, RS: Differential Equations on Fractals: A Tutorial. Princeton University Press, Princeton (2006)

22. Chilov, GE, Gurevic, BL: Integral, Measure, and Derivative: A Unified Approach. Dover, New York (1966)

23. Gordon, RA: The Integrals of Lebesgue, Denjoy, Perron, and Henstock, vol. 4. Am. Math. Soc., Providence (1994)

10.1186/1029-242X-2013-522

Cite this article as: Golmankhaneh and Baleanu: On a new measure on fractals. Journal of Inequalities and Applications 2013, 2013:522 